\title{
Cumplimiento del Protocolo de Lavado de Manos por profesionales de enfermería en un servicio de salud de la ciudad de Encarnación, marzo-julio del 2019
}

\author{
Yissel D. Bloch Melgarejo , Griselda E. Acuña Ramírez , Héctor D. Oliveira , Pedro Orué Arce \\ Universidad Católica "Nuestra Señora de la Asunción" Facultad de Ciencias de la Salud, Carrera de \\ Enfermería. Asunción, Paraguay
}

Cómo referenciar este artículo/ How to reference this article:

\begin{abstract}
Bloch Melgarejo YD, Acuña Ramírez GE, Oliveira HD, Orué Arce $\mathbf{P}$. Cumplimiento del Protocolo de Lavado de Manos por profesionales de enfermería en un servicio de salud de la cuidad de Encarnación, marzo-julio del 2019. Mem. Inst. Investig. Cienc. Salud. 2020; 18(2): 6-11
\end{abstract}

RE S U M E N

El cumplimiento del protocolo de lavado de manos evita las infecciones nosocomiales, mientras que el incumplimiento ocasiona consecuencias como el aumento de la morbimortalidad, la estancia hospitalaria, así como gastos adicionales al sistema sanitario y al usuario. Esto se puede prevenir con el lavado de manos que surgió como teoría desde la antigüedad. El trabajo de investigación fue de tipo observacional descriptivo con enfoque cuantitativo y fue realizado en 24 enfermeros de un servicio de salud de la ciudad de Encarnación con el objetivo de evaluar el cumplimiento del protocolo del lavado de manos establecido en el Manual de Prevención y Control de Infecciones asociado a la Atención de la Salud del año 2017 del Ministerio de Salud Pública y Bienestar Social. Los resultados evidenciaron un $54 \%$ de incumplimiento en la técnica de lavado de manos, un $44 \%$ de incumplimiento parcial y solamente un $2 \%$ de cumplimiento. En cuanto a los cinco momentos del lavado de manos, el $85 \%$ no cumplió con todos los momentos y un $70 \%$ no ha recibido capacitación referente al lavado de manos en los últimos 2 años. Se evidenció un alto porcentaje de incumplimiento del protocolo de lavado de manos, lo que representa un riesgo para la salud que podría subsanarse con la realización de cursos de capacitaciones o retroalimentación a los profesionales de enfermería.

Palabras clave: infecciones intahospitalarias, incumplimiento, protocolo.

\section{Compliance with the handwashing protocol by nursing professionals in a health service of the city of Encarnacion, March-J uly 2019}

\footnotetext{
A B S T R A C T

Compliance of handwashing protocol avoid nosocomial infections, while noncompliance causes consequences such as increased morbidity and mortality, hospital stay, additional cost to the health system and the patient. This can be prevented with handwashing which emerged as a theory in ancient times. This study was descriptive observational with quantitative approach and was carried out in 24 nurses at a health service in the city of Encarnacion in order to evaluate the compliance of handwashing protocol, established in the Infection Prevention and Control Manual associated to Health Care of 2017 of the Ministry of Health Care and Social Welfare. The results showed $54 \%$ of non-compliance in the handwashing technique, $44 \%$ of partial non-

Fecha de recepción: Diciembre 2019. Fecha de aceptación: Abril 2020

* Autor correspondiente: Yissel D. Bloch Melgarejo. Universidad Católica "Nuestra Señora de la Asunción" Facultad de Ciencias de la Salud, Carrera de Enfermería. Asunción, Paraguay Email: yisselbloch148@gmail.com, Institutional: fcsinvestiga.itapua@uc.edu.py, salud.uc.itapua@gmail.com.www.uci.edu.py
} 
compliance and just $2 \%$ of compliance. In relation to the five moments of handwashing technique, $85 \%$ did not follow all the steps and $70 \%$ did not receive training regarding to the hand washing technique in the last 2 years. A high percentage of non-compliance with the hand washing technique protocol was evidenced, which represents a health risk, which can be corrected with training or feedback workshops for nursing professionals.

Keywords: hospital infections, non-compliance, protocol.

\section{NTRODUCCIÓN}

Las infecciones asociadas con la atención sanitaria afectan cada año a cientos de millones de pacientes a nivel mundial y representan las principales causas de morbimortalidad. La Organización Mundial de la Salud realizo una encuesta de prevalencia en 55 hospitales de 14 países que representaban a cuatro regiones (Asia Sudoriental, Europa, Mediterráneo Oriental, Pacifico Occidental) donde el 8,7\% de los pacientes hospitalizados adquieren infecciones nosocomiales y más de 1.4 millones de personas sufren de complicaciones vinculadas con la atención sanitaria ${ }^{(1)}$.

En los países desarrollados entre el $5 \%$ y el $10 \%$ de los pacientes hospitalizados adquieren alguna infección en los Estados Unidos uno de cada 136 pacientes padece complicaciones por las infecciones contraídas en el hospital, esto equivale a 2 millones de casos y unas 80.000 muertes anuales ${ }^{(2)}$.

En los pacientes críticos el $25 \%$ desarrolla infecciones intrahospitalarias y se estima que a nivel mundial 1 de cada 4 pacientes ingresados en las unidades de terapias intensivas manifiestan infecciones nosocomiales durante su estadía. En los países con escasos recursos el porcentaje de infecciones nosocomiales es aún más importante siendo el $40 \%$ o más ${ }^{(3)}$.

Las infecciones intrahospitalarias son prevenibles mediante una técnica sencilla, económica y muy eficaz que es el lavado de manos, esto surge como teoría desde la antigüedad a mediados del siglo XIII gracias a un médico húngaro, Ignaz Semmelweis quien descubrió que las enfermedades contraídas en los hospitales eran propagadas por las manos y esto se podía evitar lavando las manos antes de brindar atención a los enfermos, desde aquel entonces el lavado de manos quedo establecida como técnica universal. A base de esto en1975 y 1985 el Centro para la Prevención y el Control de Enfermedades en Atlanta Estados Unidos público reglamentos que demostraban el correcto procedimiento para realizar el lavado de manos y designó elementos necesarios para su ejecución, como el uso de antisépticos ${ }^{(4)}$.

La Organización Mundial de la Salud en el año 2002 en la 55a asamblea estableció la promoción mundial sobre la seguridad del paciente reforzando los sistemas de seguridad y control por medio de guías y protocolos sobre el lavado de manos. EI incumplimiento conlleva a la aparición de enfermedades infectocontagiosas y con ello, morbimortalidad, internación hospitalaria prolongada, cargas financieras adicionales y estrés a los pacientes y familiares ${ }^{(5)}$.

Los riesgos producidos por el incumplimiento del lavado de manos en los centros de salud, especialmente en las unidades de cuidado intensivo, son las infecciones nosocomiales que se presentan de manera frecuente, por tal motivo se justifica evaluar el cumplimiento del protocolo de lavado de manos en un servicio de salud de la cuidad de Encarnación para evidenciar el cumplimiento del mismo como una garantía de prevención de enfermedades infectocontagiosas.

\section{MATERI ALES Y MÉTODOS}

Este es un estudio observacional descriptivo con enfoque cuantitativo

Como materiales para la higiene de las manos se utilizó jabón líquido (yodopovidona al $7,5 \%$ y clorhexidina al $4 \%$ ), y para el secado de las manos toalla de papel simple o doble. Como instrumento de recopilación de datos se utilizaron tres planillas A, B y C, la planilla $A$ con tres indicadores referidos a la forma de realización del lavado de manos, la planilla B con cinco indicadores sobre los momentos del lavado de manos y la planilla $\mathrm{C}$ se utilizó para recabar datos respecto a las capacitaciones recibidas durante los últimos dos años. La recopilación de datos se realizó de marzo a julio, 
toda la semana y en los tres turnos, mañana, tarde y noche y entre 2 a 3 observaciones por cada enfermero dependiendo de los turnos y obteniendo 54 observaciones totales.

En la planilla A se calificó el cumplimiento de los pasos establecidos en la técnica del lavado de manos, observando tres indicadores los cuales fueron sobre el cumplimiento total de los pasos, el incumplimiento y cumplimiento parcial de los pasos. En la planilla B se evaluó el cumplimiento de la técnica del lavado de manos en los cinco momentos indicados por la Organización Mundial de la Salud establecido en el Manual de Prevención y Control de Infecciones asociadas a la Atención de la Salud, año 2017 del Ministerio de Salud Pública y Bienestar Social ${ }^{(6)}$, como así también preguntas acerca de capacitaciones sobre dicha técnica que se evidencian en la planilla $C$.

Para realizar la observación se tuvieron en cuenta los pasos establecidos en el Manual de Prevención y Control de Infecciones asociadas a la Atención de la Salud, año 2017 del Ministerio de Salud Pública y Bienestar Social, donde se indican los respectivos pasos a seguir: abrir la llave del agua con la mano dominante y mojar vigorosamente las manos, aplicar jabón antiséptico en la palma de la mano, friccionar toda la superficie de las manos durante 10 a 15 segundos en siguiente orden; palma y dorso con movimientos circulares, espacios interdigitales, pulgar y dedos con movimientos rotatorios, uñas y muñeca. Posteriormente, enjuagar vigorosamente, secar las manos con toallas de papel descartables y cerrar la llave de agua con toalla descartable y desechar ${ }^{(6)}$.

Para la observación de los momentos del lavado de manos se consideró al ingreso en el servicio, antes del contacto con el paciente, antes de realizar una tarea aséptica, después de realizar una tarea aséptica y antes de salir del servicio. Y para recabar datos referentes a las capacitaciones realizadas en los últimos dos años se entablaron preguntas al respecto.

Los datos recopilados fueron cargados en planillas Excel Office 2016 y procesados estadísticamente para obtener gráficos de barras y circulares conteniendo porcentaje de los indicadores específicos.

\section{RESULTADOS}

Se trabajó con 24 enfermeros a cargo de un servicio de salud de la ciudad de Encarnación con predominio del sexo femenino del $62 \%$, en cuanto a la edad el $38 \%$ tenía entre 25 a 30 años, el $29 \%$ entre 31 a 35 años, el $21 \%$ entre 36 a 40 años y el $12 \%$ entre 41 a 45 años. Referente a la antigüedad en el ejercicio de la profesión el $46 \%$ posee de 0 a 5 años, el $29 \%$ de 6 a 10 años y el $25 \%$ de 11 a 15 años (Figura $1)$.

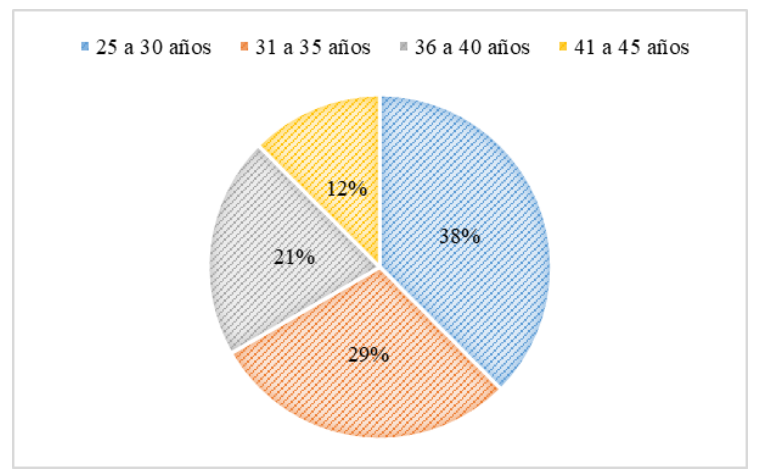

Figura 1: Porcentaje de edad de los profesionales de enfermería.

En la Figura 2 se observan el porcentaje de cumplimiento de la técnica de lavado de manos, donde el $54 \%$ de los observados no cumplieron con la técnica del lavado de manos, el $46 \%$ cumplieron con la técnica, de los cuales solamente el $2 \%$ cumplieron adecuadamente con los pasos indicados en la técnica y un $44 \%$ lo hicieron parcialmente. 


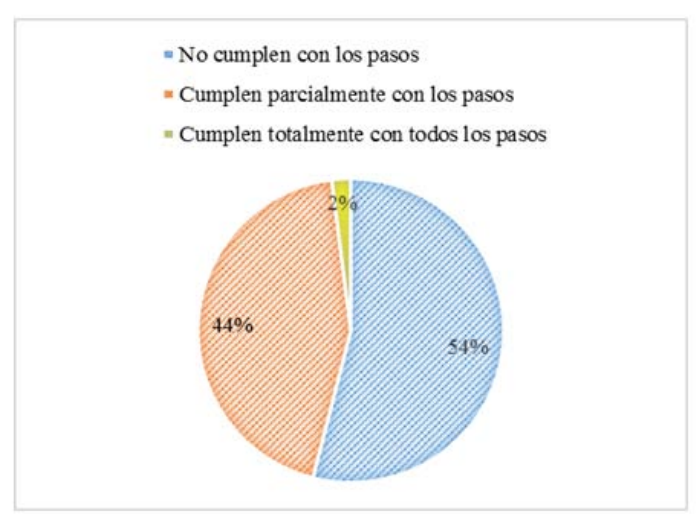

Figura 2: Porcentaje de cumplimiento de la técnica de lavado de manos.

Referente a la realización del lavado de manos en sus cinco momentos, la Figura 3 muestra que el 35\% realizaron al ingreso del servicio, antes de realizar una tarea aséptica y después de salir del servicio, el $22 \%$ realizaron al ingreso del servicio, antes del contacto con el paciente, después de realizar una tarea aséptica y antes de salir del servicio, el $20 \%$ al ingreso del servicio, antes de realizar una tarea aséptica, después de realizar una tarea aséptica y antes de salir del servicio, solamente el $15 \%$ cumplió con los momentos del lavado de manos determinadas en el protocolo, es decir, lo realizaron al ingresar al servicio, antes del contacto con el paciente, antes de realizar una tarea aséptica, después de realizar una tarea aséptica y antes de salir del servicio y el $8 \%$ realizaron el lavado de manos después de realizar una tarea aséptica y antes de salir del servicio.

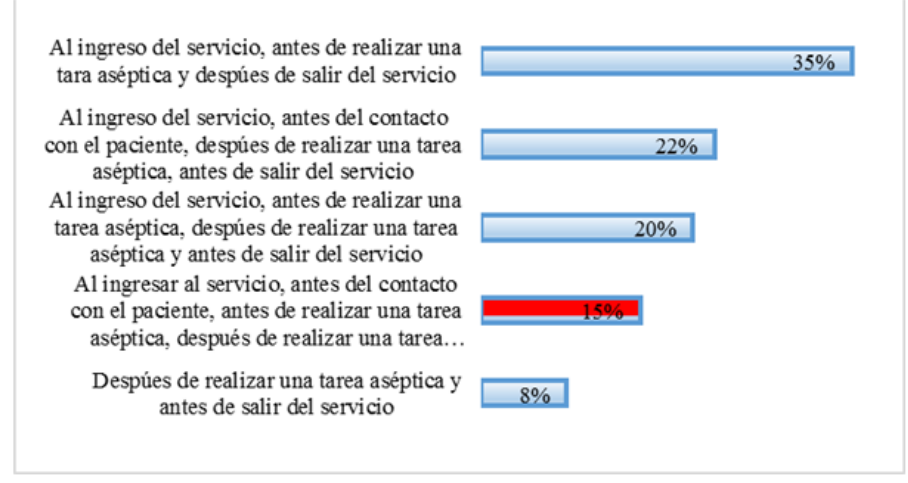

Figura 3: Porcentaje de la realización del lavado de manos en sus cincos momentos.

Se puede apreciar en la Figura 4 que el $70 \%$ de los enfermeros no recibieron capacitaciones en los últimos 2 años referido al protocolo del lavado de manos y el $30 \%$ si lo recibieron.

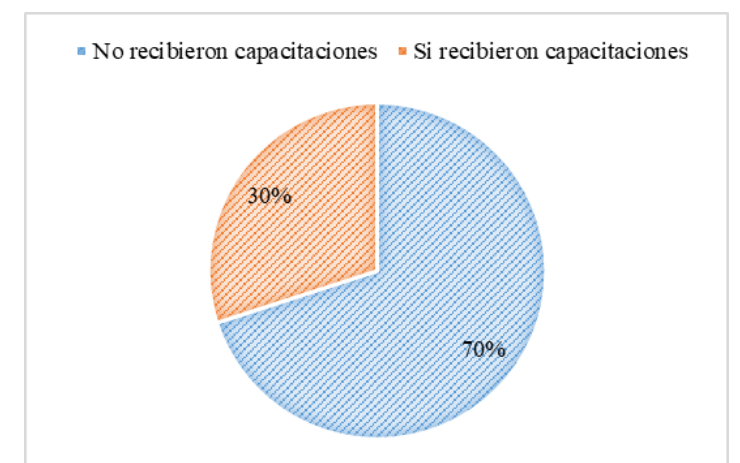

Figura 4: Porcentajes de capacitación recibida en los últimos 2 años respecto al protocolo de lavado de manos. 


\section{DISCUSIÓN}

Respecto a la técnica de lavado de manos por los enfermeros de un servicio de salud de la cuidad de Encarnación, los resultados arrojaron un 54\% de incumplimiento del protocolo de lavado de manos, estudios realizados por Riveros Pérez, Zambrano y Amado en una clínica privada de Colombia en el 2012 han determinado el cumplimiento del lavado de manos menos del $50 \%$ y comprobaron que el motivo se debió a la falta de conocimiento de los protocolos ${ }^{(7)}$.

Los investigadores Sares y Solis en 2009 hacen referencia a que el incumplimiento del lavado de manos en un hospital de Ecuador oscila entre el $43 \%$ y el $60 \%$, concluyendo que los profesionales lavan sus manos la mitad de las veces de las que está indicada y con menor duración a la recomendada ${ }^{(8)}$.

Otro análisis estadístico hecho por De Vita y colaboradores en una Unidad de Terapia Intensiva de Argentina demostraron que el incumplimiento del lavado de manos fue de $41 \%$, porcentaje casi estimativo a lo nuestro, en donde se pudo comprobar un $54 \%$ de incumplimiento. También señalaron que el cumplimiento del lavado de manos fue de $29 \%$ a $87 \%$, nuestros resultados obtenidos al respecto fueron casi similar consiguiendo un $46 \%$ de cumplimiento, de los cuales solamente el $2 \%$ efectuó todos los pasos establecidos para la realización del lavado de manos y el $44 \%$ lo realizo bajo la denominación de cumple parcialmente. Por otro lado, comprobaron que un $47 \%$ de los observados se lavaban las manos antes del contacto con el paciente y el $70 \%$ después del contacto con el paciente, este último dato hace referencia a que el profesional prevalece el lavado de manos después de la atención al paciente por seguridad propia ${ }^{(9)}$.

Por otro lado, una investigación hecha por Saldarriaga y colaboradores en un hospital de Perú en el 2015, identificaron que el lavado de manos posee mayor adherencia en los momentos 1 y 4 , es decir antes del contacto con el paciente y después del contacto con el paciente, esta situación es semejante a lo observado en el servicio de salud de la cuidad de Encarnación donde priorizaban el lavado de las manos después del contacto con el paciente ${ }^{(10)}$.

Un estudio realizado en un hospital de Colombia por Henao y Sierra en el 2014 a un grupo de alumnos de enfermería y medicina sobre evaluación en la calidad de aplicación de la técnica del lavado de manos constato que no se lavaban las manos correctamente, haciendo énfasis en la parte educativa como factor predisponente y primordial en la aplicación correcta de la técnica ${ }^{(11)}$.

Zamudio y otros investigadores manifestaron que en un hospital de tercer nivel de México las capacitación sobre el lavado de manos son denegadas porque se considera una técnica habitual, por otro lado Pittet y colaboradores argumentaron mediante intervenciones donde se incluyó capacitaciones se consiguió una adherencia del lavado de manos y a su vez una reducción importante en la tasa de infecciones, esto se tomó como base en la realización de la Guía de Higiene de Manos en el cuidado de la salud lanzado por la Organización Mundial de la Salud en el año $2009^{(12)}$.

Los datos obtenidos en el servicio de salud de la ciudad de Encarnación referente a cursos de capacitaciones sobre el lavado de manos, se comprobó que el profesional ha tenido una baja participación al respecto y solamente el $30 \%$ acudieron a curso de capacitaciones en los últimos dos años.

Se evidencia el incumplimiento del protocolo de lavado de manos por los profesionales de enfermería de un servicio de salud de la cuidad de Encarnación, lo que pone en riesgo la salud de los pacientes ya que ello incrementa las tasas de infecciones asociadas a la atención sanitaria/ infecciones intrahospitalarias.

Es necesario monitorear de forma sistemática el cumplimiento del protocolo y aplicar las observaciones y sanciones correspondientes acorde a lo estipulado en el reglamento de salud. Propiciar cursos de capacitaciones o retroalimentación abordando el tema con mayor compromiso y profesionalismo dado que según datos oficiales el de la Organización Mundial de la Salud hasta un $70 \%$ de los profesionales de salud no realizan las prácticas recomendadas de higiene de las manos. 


\section{REFERENCI AS BI BLI OGRAFI CAS}

1. Organización Mundial de la Salud. Directrices de la OMS sobre Higiene de las Manos en la Atención Sanitaria. 2005.

2. Romero Mora KA, Soto Ramírez LE. Primer paso en el control de las infecciones relacionadas a la atención médica: Lavado de manos. Revista Médica Sur. 2008 Julio; 15(3): 214-18.

3. Guerrero López LM, Barajas Medina H, Caudillo Cisneros C, López Nava SM, Escalante Piña O. Capacitación y Adherencia al lavado de manos y su efecto en la infección nosocomial. Revista Mexicana de enfermería. 2012; 20(3): 157-65.

4. Padron ER, Companoni Landin FA, Rosales Reyes SA. Apuntes Históricos sobre el Lavado de las Manos. Revista Cubana de Estomatología. 2015; 52(2): 21726.

5. Oliva LM, Rosa L. El Conocimiento $Y$ Aplicación Del Correcto Lavado De Manos Del Profesional De Enfermeria Y La Influencia En La Recuperación De Los Pacientes En EI Servicio De Cirugía General Del Hospital Barranca Cajatambo Junio Julio 2017. Universidad Autónoma de Ica [Internet]. 16 de mayo de 2018 [citado 11 de agosto de 2020]; Disponible en: http://localhost/xmlui/handle/auton omadeica/243

6. Ministerio de Salud Pública y Bienestar Social. Manual de Prevención y Control de Infecciones Asociadas a la Atención de la Salud. 2017.

7. Riveros Pérez, Efraín; Zambrano, Paula; Amado, Paula. Adherencia a las guías de higiene de manos en cuidado intensivo: el caso de una clínica privada Medicina UPB,2012. 31(2): 127-34

8. Sares Carrión VG SSJ . Cumplimiento del lavado de manos por parte del personal de salud del servicio de emergencia del Hospital José Carrasco Arteaga, Cuenca- Ecuador 2008. Cuenca. Universidad de Cuenca; Facultad de Ciencias Médicas . Escuela de Medicina. [Tesis doctoral]: 2009.

9. De Vita V, Weisburd G, Beltramino D, Bussi E. Conocimiento, actitudes y prácticas del personal de salud relacionados con el lavado de manos clínico en una unidad de cuidados intensivos. Revista Médica Rosario. 2014; 80: 105-16.

10. Saldarriaga Sandoval LJ, Barreto Boulangger JF, Córdova García DS. Adherencia al lavado de manos en personal de salud del Hospital Regional José Alfredo Mendoza Olavarria II-2 de Tumbes. Revista Iberoamericana en Educación e Investigación en Enfermería. 2016; 6(4): 55-61.

11. Henao González S, Sierra P. Asociación entre Adherencia a Higiene de Manos y Características Sociodemográficas, Laborales y de Locus de Control en los Profesionales de la Salud de la IPS Universitaria sede León XIII. 2014.

12. Saldarriaga Sandoval LJ, Barreto Boulangger JF, Córdova García DS. Adherencia al lavado de manos en personal de salud del Hospital Regional José Alfredo Mendoza Olavarria II-2 de Tumbes. Revista Iberoamericana en Educación e Investigación en Enfermería. 2016; 6(4): 55-61.

13. Zamudio Lugo I, Meza Chávez A, Martínez Sánchez $Y$, Miranda Novales MG, Espinosa Vital JG, Rodríguez Sing R. Estudio Multimodal de Higiene de Manos en un Hospital Pediátrico de Tercer Nivel. Boletín Médico del Hospital Infantil de México. 2012; 69(5): 384-90. 